\title{
Enlarge Medical Image Using Line-column Interpolation (LCI) Method
}

\author{
Jufriadif Na`am, Julius Santony, Yuhandri, Sumijan, Gunadi Widi Nurcahyo \\ Faculty of Computer Science, Universitas Putra Indonesia YPTK Padang, 25221, Indonesia
}

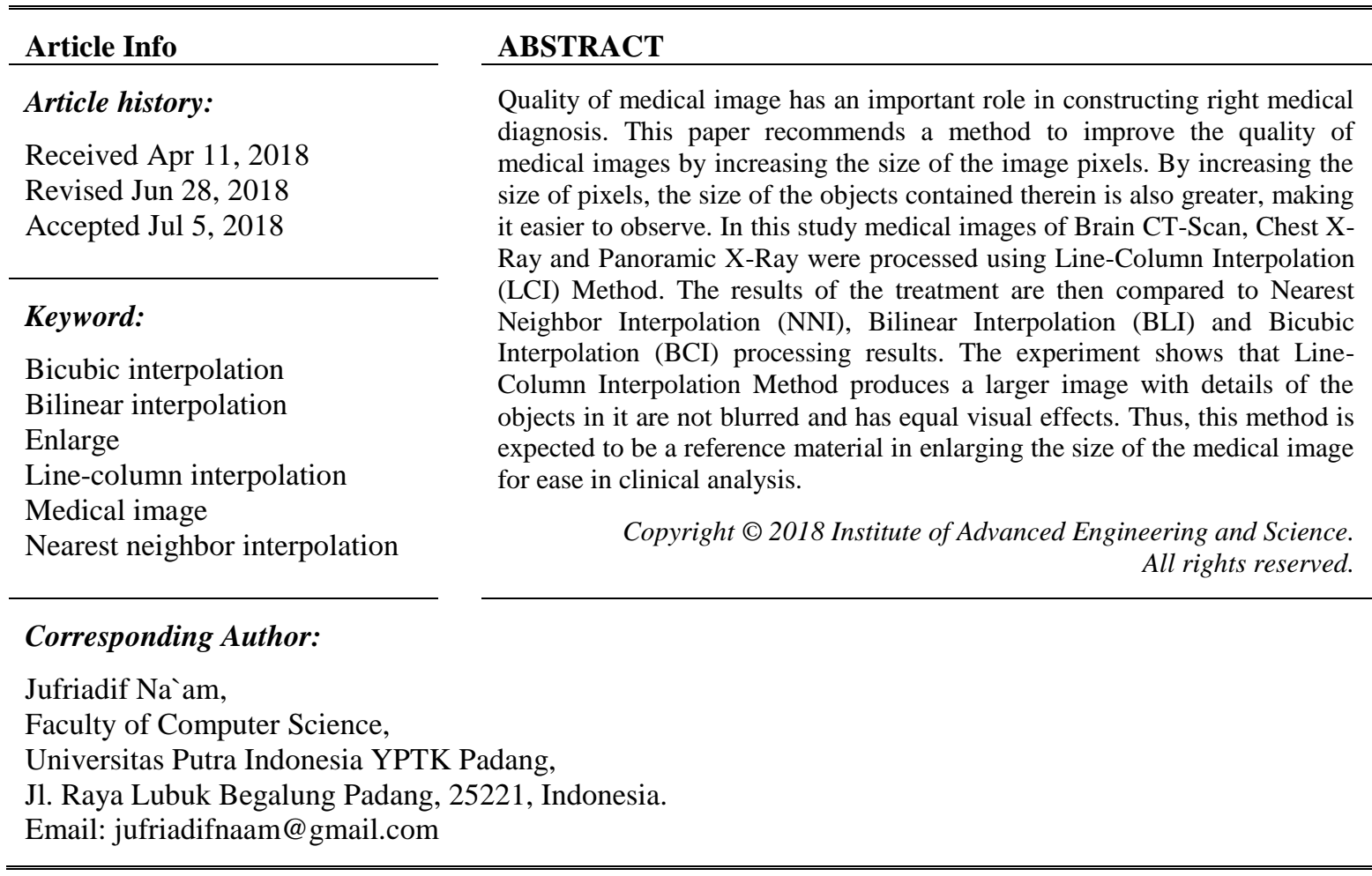

\section{INTRODUCTION}

Medical images are a clinical need to understand diseases in care and treatment [1][2]. Good imaging ease medical personnel to produce more precise clinical trials. However, to obtain a good image, a sophisticated technology which is usually expensive is to be required. The procurements of such equipment is charged to the patient [3]. Therefore, a better information technology approach is needed to diagnose diseases [4]. One such information technology approach is the medical image. Medical images can be used for early diagnosis in accurately identifying dysfunctional objects in the human body [5].

Some steps are required in the processing of medical images for better influence of clinical outcomes. The necessary step is to improve the accuracy of the image first. This improvement makes the diagnosis accurate. The task of refining the medical image can be supported by increasing the size of the image, but still paying attention to the preservation of the details of the particular image [6]. The enlarged image contains problems such as loss of contrast and the detail becomes blurred.

Besides, enlarging the image size is also a fundamental process. Larger sizes should be able to retain important content in imagery as well as visual effects in general. The main disadvantages that often occur are local structural damage or visual effects [7]. Increasing the image size is required to increase the size of the initial image pixels. The image enlargement adds new pixels and narrows the sampling interval of the image to the pixel value interpolation. The value of this additional pixel is determined by the interpolation (the value of its neighboring pixels). Existing interpolation methods are Nearest Neighbor Interpolation (NNI), Bilinear Interpolation (BLI) and Bicubic Interpolation (BCI) [8]. In this research, we propose a Line-column Interpolation Method (LCI) as a new method. This method is tested to enlarge the size of the medical image. 
Larger image sizes produce larger objects by retaining the value of their local structure. Thus, the result of this magnification makes better observation to the objects in the image.

The LCI method is tested using medical image of Brain CT-Scan, Chest X-Ray and Panoramic XRay in Matlab software. The same data are also tested in the same way by implementing NNI, BLI, and BCI methods [9]. Fundamental principles that need to be fulfilled by image enlargement is that the composition ratio, namely the value and position of pixel must be the same as the original image can be tested using a histogram.

Several studies on image size enlargement were Suetake et al [10] conducted a study to accelerate image enlargement using BCI method against empty pixels using back projection technique. Besides that, Zhang et al [11] performed image expansion using the nearest quadratic polynomial precision (NNI) method. Image enlargement results were also tested for edge detection; such as study by Zhong et al [12] conducted a study of retaining the border of an object against an enlarged image using the BCI method.

There will be additional pixels in image enlargement. The value of these additional pixels may be empty, missing or not synchronized (exact). Olivier et al. [13] conducted a study in the determination of missing pixel values in enlarging high-resolution images using the nearest neighbor method. Bashar et al. [14] conducted a study using the bilinear interpolation method to estimate incorrect pixel values in face image recognition. Kai-yu et al [15] designed a method to enlarge high-quality and fast image display based on FPGA (Field Programmable Gate Array) using the Bicubic method. Gopinathan et al [16] conducted a study to remove noise (denoise) by applying the BLI method to the image portion having low frequency. The limited number and different emphasizes of those previous studies, we conducted a study to enlarge the image by using Line-column Interpolation Method on grayscale medical images.

\section{RESEARCH METHOD}

The objects contained in the medical image must be observable. Errors in the observation of these objects can lead to misdiagnosis. This error complicates the patient's care and treatment process. To facilitate this observation, it is necessary to enlarge the size of the object. In this study, a method of enlarging objects in medical images is proposed where the sequence of the process can be seen in Figure 1.

The process stages of this study consist of 4 (four processes to produce an enlarged image file). Every step of enlargement process produces image size 2 times the size of the input image pixel. The activation process can repeateadly be done until the maximum size of memory type data used is no longer sufficient (out of memory). Illustration of LCI method can be seen in Figure 2.

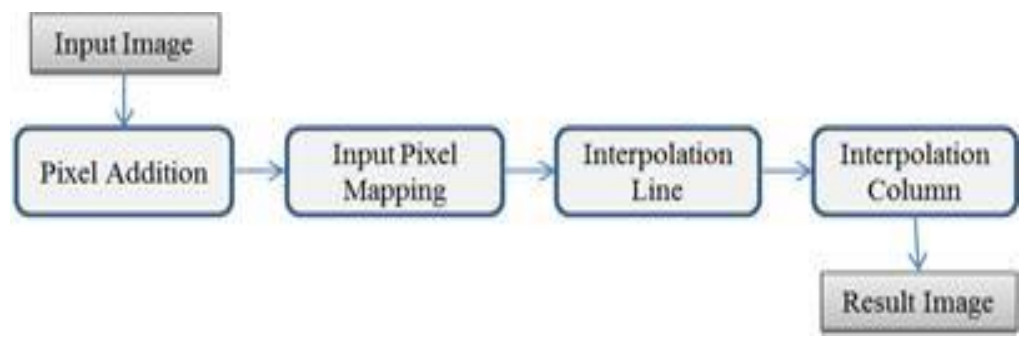

Figure 1. Stage of process on CLI method

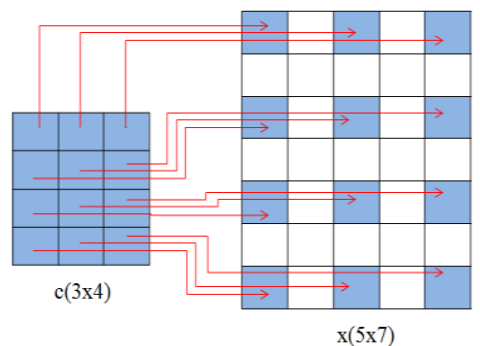

(a)

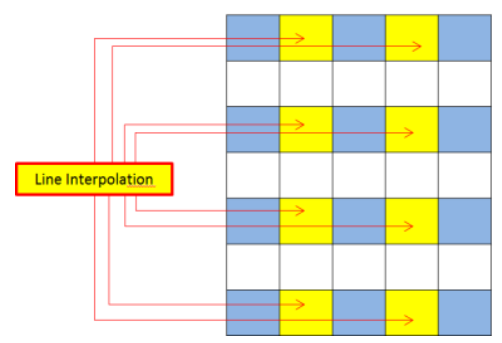

(b)

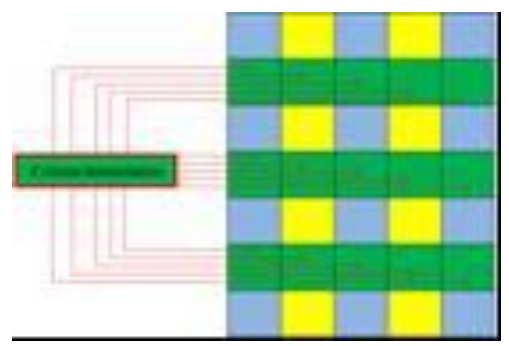

(c)

Figure 2. Illustration of LCI, (a) Add and input the pixel mapping, (b) Line Interpolation, (c) Column Interpolation 


\subsection{Input Image}

The input images processed in this research are obtained from radiology department of Central General Hospital (RSUP) Dr. M. Djamil Padang. All of the images are in the form of the grayscale image where details about the data can be seen in Table 1 . This input image was used in previous studies with a specific purpose [17-18].

Table 1. Input Image Specification

\begin{tabular}{llc}
\hline \multicolumn{1}{c}{ Category } & \multicolumn{1}{c}{ Format } & Quantity \\
\hline Computed Tomography Scan (CT Scan) & Windows bitmap (bmp) & 5 \\
Chest X-Ray & Joint Photographic Experts Group (jpg) & 42 \\
Panoramic X-Ray & Portable Network Graphics (png) & 27 \\
\hline
\end{tabular}

\subsection{Pixel Addition} equation is:

Image enlargement is done by adding new pixels. So the image size is enlarged to $\mathrm{n}$ times, the

$$
f(z)=n . c
$$

where: $f(z)$ : image enlargement

$\mathrm{n}$ : image size proliferation

c: input image

For each enlargement process in the testing phase, addition of as many pixels as the previous image is applied. So the image size becomes 2-fold from the previous size. An example illustration of the addition of pixels can be seen in Figure 2(a). If kc initializes the number of initial image columns and, bc initializes the number of rows then the number of image enlargement columns becomes $\mathrm{kx}$ and the rearing row becomes bx. Then the equations of many rows and columns of magnification image are as follows:

$$
\begin{aligned}
& k x=k c+k c-1 \\
& b x=b c+b c-1
\end{aligned}
$$

\subsection{Input Pixel Mapping}

To map an initial pixel image value with a magnified image based on pixel position, the following equation pixel input is used:

$$
z_{(k, b)}=c_{(k+k-1, b+b-1)}
$$

\subsection{Line Interpolation}

Line Interpolation is the process of charging against empty pixels using the closest value in a row. The position of the processed row is interrupted by an existing pixel value. The illustration of the line interpolation can be seen in Figure 2(b). The value of the empty pixels between the lines is specified using the following formula:

$$
z_{(k, b)}=\frac{z_{(k-1, b)}+z_{(k+1, b)}}{2}
$$

\subsection{Column Interpolation}

Once the blank pixel value in a row filled, columns are interpolated. Interpolation of columns is the process of charging against empty pixels using the closest value in a single column. The position of the processed column is interrupted by an existing pixel value. Illustration of line interpolation can be seen in Figure 2(c). The value of empty pixels between columns are calculated using the following formula:

$$
z_{(k, b)}=\frac{z_{(k, b-1)}+z_{(k, b+1)}}{2}
$$




\section{RESULTS AND ANALYSIS}

Tests conducted on this method is the cropped medical image data with manual [17]. This is essential to eliminate unneeded areas in the analysis. One image of each data format presented in this paper can be seen in Figure 3. Prior to the process of image enlargement, an image area that would become the object of observation was taken. The process of taking this area was done by cutting (crop). Image of processing result can be seen in Table 2 .

Based on the test results in Table 2, it can be observed that the image result has relatively the same size. The difference is, the image results of the proposed method has only a 1-pixel column and 1-pixel row less than those of the NNI, BLI and BCI methods. This deficiency is caused by the addition of pixels only on the sidelines of the original pixels. However, this 1-pixel difference can still be ignored because it is only a deficiency in the final row or column position and not the position of the object to be observed in the medical image.

Table 2. Two Times Enlargement Result (The Size is $25 \%$ of the Original Size)

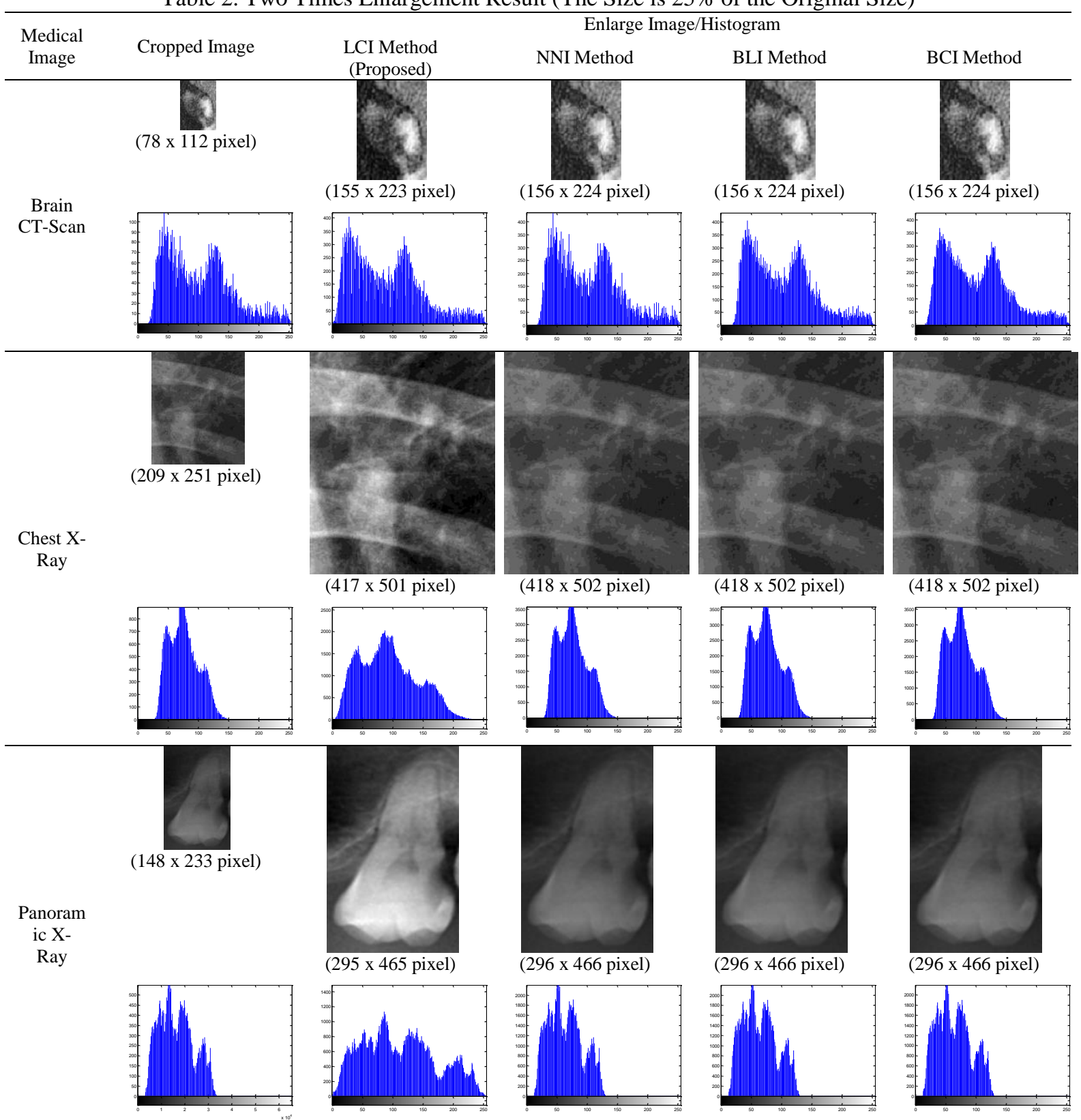




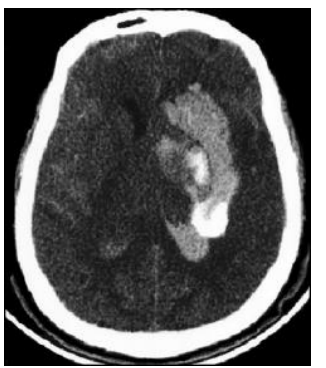

(a)

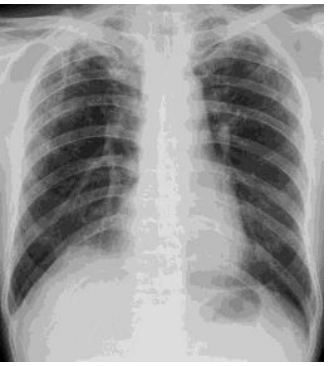

(b)

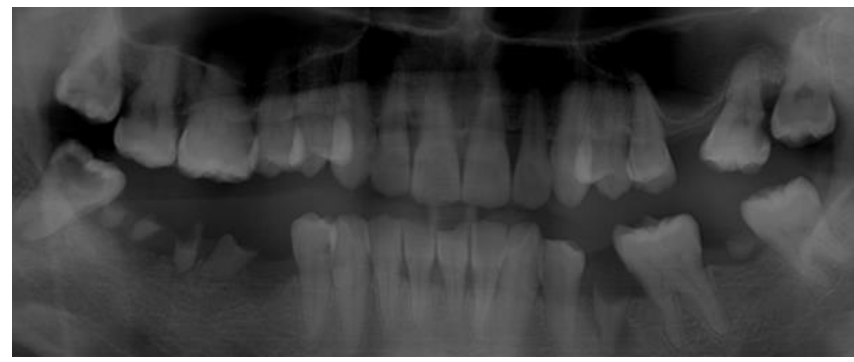

(c)

Figure 3. Image test (a) Brain CT-Scan, (b) Chest X-Ray, (c) Panoramic X-Ray

The form of objects contained in the image of the results can be well observed. This can be seen from the fluctuation of the histogram graph, particularly through the relatively similar spread of grey values. Principally, the difference between objects on the image of the proposed method appears more sharply. The spread of more gray values causes the sharpness of this object. Here is the result of the enlargement process to 4 times from the initial image to Brain CT-Scan image. The results of the comparison can be seen in Figure 4.

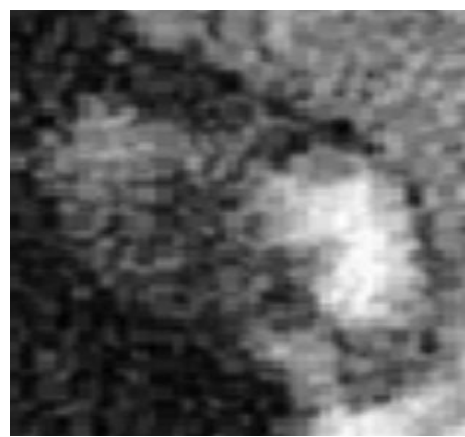

(a)

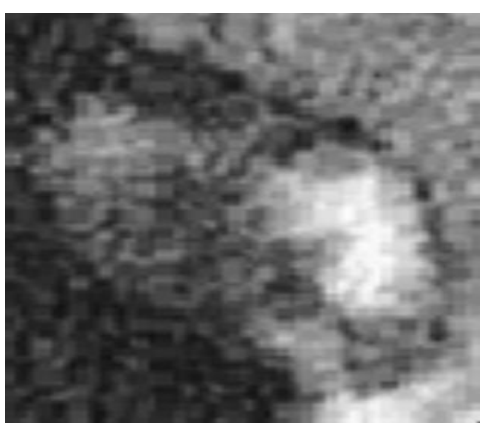

(c)

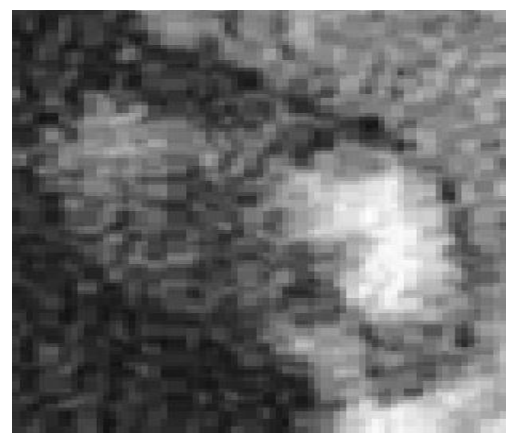

(b)

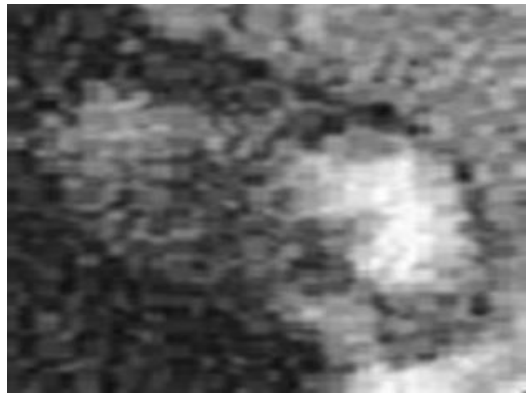

(d)

Figure 4. 4 times Brain CT-Scan Image enlargement (in original size), (a) Proposed method, (b) NNI method, (c) BLI method, (d) BCI method

The pixel size at 4 times magnification results for the proposed method is $309 x 445$ pixels, while the other method has the same size, which is $312 \times 448$ pixels. This $4 \times 3$ pixel difference is not very influential, where:

$$
\frac{3 \times 4}{312 \times 448}=\frac{12}{139776}=0.000086
$$


0.000086 is the relatively small difference between image size in the proposed method compared to the image size of existing methods. In Figure 4 can also be observed that the difference between the new pixel values on the proposed method and the underlying pixels of the initial image is not so conspicuous. Therefore, the details of the objects in the image are still the same. The differences of this proposed method to existing methods (NNI, BLI and $\mathrm{BCI}$ ) are additional pixel values closer to the value of neighboring pixels so the detail view is not blurred.

\section{CONCLUSION}

From the test results of the medical image can be seen that the image enlargement using LCI can increase the size of the original image. Each stage in the process produces an image size two times larger than the original image. Thus, the objects contained in the image result are larger and sharper. This study indicates that the LCI method can facilitate the observation of objects in the medical image in the process of medical analysis.

\section{ACKNOWLEDGEMENTS}

We would like to thank for Director and all staff of Department of Radiology, Dr. M. Djamil Center General Hospital, Padang, which has allowed the use of the data in this study.

\section{REFERENCES}

[1] B. I. Reiner, et al, "The Clinical Imperative of Medical Imaging Informatics," Journal of Digital Imaging, vol.22, no.4, pp. 345-347, 2009.

[2] S. Bhargava and A. Somkuwar, "Evaluation of Noise Exclusion of Medical Images using Hybridization of Partical Swarm Optimization and Bivariate Shrinkage Methods," International Journal of Electrical and Computer Engineering (IJECE), vol.5, no.3, pp.421-428, 2015.

[3] A. T. B. Alex and K. T. Ricky, Medical Imaging Informatics, Springer New York Dordrecht Heidelberg, London, pp.3, 2010.

[4] S. Pohoata and A. Graur, "HDTV System for Parkinson's Disease Diagnosis," Advances in Electrical and Computer Engineering, vol.13, no.2, pp.91-96, 2013.

[5] F. Malik, et al, "An Ensemble of Classifiers based Approach for Prediction of Alzheimer's Disease using fMRI Images based on Fusion of Volumetric, Textural and Hemodynamic Features," Advances in Electrical and Computer Engineering, vol.18, no.1, pp.61-70, 2018.

[6] R. M. Kokate and V. K. Shandilya, "Enhancement Techniques of Medical Images-A Review," International Journal of Advanced Research in Computer and Communication Engineering, vol.5, no.2, pp.533-535, 2016.

[7] W. Dong, et al, "Optimized Image Resizing Using Seam Carving and Scaling," ACM Transactions on Graphics, Association for Computing Machinery, vol.29, no.5, pp.1-10, 2009.

[8] L. Seongsu and J. Gwanggil, "Lagrange Method for Upsampling," Advanced Science and Technology Letters, vol.80, pp.9-13, 2015.

[9] MathWorks: Image Processing website, https://www.mathworks.com/matlabcentral/answers/14103-image-processing

[10] N. Suetake, et al, "Fast image enlargement using predefined codebook and eigenspace backprojection for lost pixel," Optical Review, vol.18, no.6, pp.423-429, 2011.

[11] F. Zhang, et al, "Enlarging image by constrained least square approach with shape preserving, "Journal of Computer Science and Technology, vol.30, no 3, pp.489-498, 2015.

[12] X. Zhong, et al, "A novel adaptive image zooming scheme via weighted least-squares estimation," Frontiers of Computer Science, vol.9, no.5, pp.703-712, 2015.

[13] R. Olivier and C. Hanqiang, "Nearest Neighbor Value Interpolation," (IJACSA) International Journal of Advanced Computer Science and Applications, vol.3, no.4, pp.1-6, 2012.

[14] F. Bashar, et al, "Face Recognition using Similarity Pattern of Image Directional Edge Response," Advances in Electrical and Computer Engineering, vol.14, no.1, pp.69-76, 2014.

[15] L. Kai-yu, et al, "The application of B-spline based interpolation in real-time image enlarging processing," The 2014 2nd International Conference on Systems and Informatics (ICSAI 2014), Shanghai, pp.823-827, 2014.

[16] S. Gopinathan, et al, "Wavelet and FFT Based Image Denoising Using Non-Linear Filters," International Journal of Electrical and Computer Engineering (IJECE), vol.5, no.5, pp.1018-1026, 2015.

[17] J. Na`am, et al, "Detection of proximal caries at the molar teeth using edge enhancement algorithm," International Journal of Electrical and Computer Engineering (IJECE), vol.8, no.5, pp. 3259-3266, 2018. 


\section{BIOGRAPHIES OF AUTHORS}
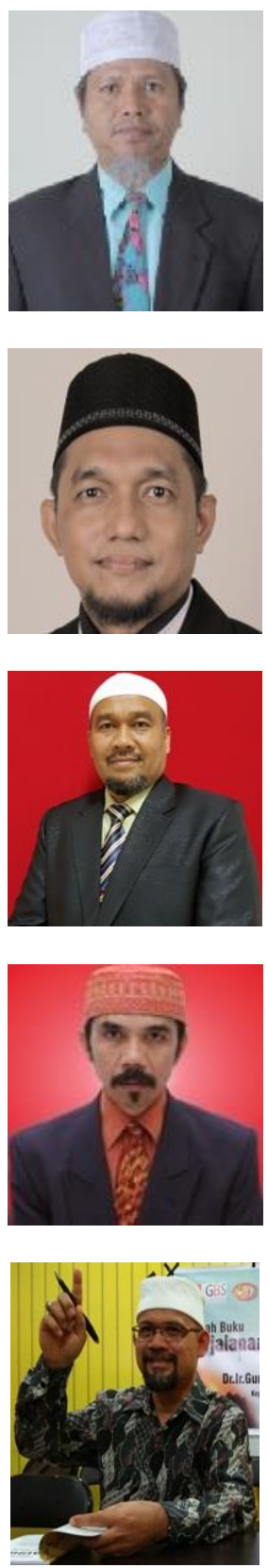

Jufriadif Na`am was born in Padang, West Sumatra - in 1967. He is an Associate Professor in Faculty of Computer Science, Universitas Putra Indonesia YPTK. He received the Bachelor Degree in Informatics Management and Master Degree in Information Tecnology in 1994 and 2006 from Universitas Putra Indonesia YPTK. Moreover, he completed his Doctorate of Information Technology as Informatics Medical Image expertise from Gunadarma University in January 2017. He has member of IEEE (94313333). Scopus Id is 57189371499. Currently, he has been researching on Algorithms and Informatics Medical Image. E-mail: jufriadifnaam@gmail.com

Julius Santony was born in Padang Panjang, West Sumatera, Indonesia, on July 29th 1973. His Department is Computer Science, Universitas Putra Indonesia YPTK Padang, Wes Sumatera Indonesia and $\mathrm{He}$ is an Associate Professor. He graduated the Bachelor Degree in 1996 in Informatics Management form Gunadarma University and Master Degree in 2006 in Information Tecnology from Universitas Putra Indonesia YPTK. Then, he graduated the Doctorate Degree of Information Technology as Medical Image expertise from Gunadarma University in December 2015. Scopus Id is 57189362021. Sustainably, he has been researching on Image Processing Algorithm for Medical Images. E-mail: juliussantony@ yahoo.co.id

Yuhandri was born in Tanjung Alam on May 15. He is an Assistant Professor in Faculty of Computer Science, Universitas Putra Indonesia YPTK. He received the Bachelor Degree in Informatics Management and Master Degree in Information Tecnology in 1992 and 2006 from Universitas Putra Indonesia YPTK. Moreover, he completed his Doctorate of Information Technology as Informatics Medical Image expertise from Gunadarma University in April 2017. $\mathrm{He}$ is a lecturer at the Faculty of Computer Science, Universitas Putra Indonesia YPTK. Scopus Id is 57193430920. E-mail: yuyu@ upiyptk.ac.id

Sumijan was born in Nganjuk on May 7 1966. He received the Bachelor Degree in Informatics Management in 1991 from Universitas Putra Indonesia YPTK, Master of Information Technology in 1998 from University Technology Malaysia (UTM). He completed has Doctorate of Information Technology as Medical Image Expertise from Gunadarma University in December 2015. He is member of ACM (23145751). Scopus Id is 57194787076. E-mail: soe@upiyptk.org

Gunadi Widi Nurcahyo was born in Temanggung, 14 March 1969. He was graduated Bachelor degree in Informatics Management at Universitas Putra Indonesia YPTK Padang in 1992. He completed his Master and PhD in Computer Science at Universiti Teknologi Malaysia in 2003. Scopus Id is 57200563356. E-mail: gunadiwidi@yahoo.co.id 